\title{
Extending the healthspan
}

\section{Linda Partridge examines studies on preventative medicine for the ageing.}

$\mathrm{T}$ he chances of living to old age are higher than ever in many parts of the world. So, particularly in developed countries, health-care systems are struggling to cope with the silver tsunami' of elderly people with clusters of diseases for which age is the main risk factor, including cancer, diabetes, cardiovascular disease, sarcopenia and dementia. Fortunately, the opportunity is at hand to transform the landscape and keep people in better health as they age.

Aging is a collection of articles edited by gerontologists Jay Olshansky, George Martin and James Kirkland. It explores the potential to extend human health by drawing on discoveries about the biology of ageing. The overall coverage is US-centric, and would have benefited from more cutting-edge basic science and human demography from the rest of the world. But the message is clear: it is time to begin the revolution in medical approaches to ageing-related disease and late-life health.

In lab animals, Aging tells us, simple genetic and environmental interventions can increase healthy lifespan substantially. A restricted diet can protect ageing rodents and rhesus monkeys from most impairments and diseases. Genetic alterations to the signalling networks that sense and respond to nutrients and to other inputs can have similar effects. These interventions tamp down the changes that characterize ageing and that lead to pathology, including chronic inflammation, cellular senescence, damage to macromolecules and decline in stem-cell function.

An emerging consensus thus regards ageing as composed of modifiable subsyndromes. These could be targeted with drug combinations and environmental changes to produce a broad-spectrum, preventive medicine for multiple ageing-related diseases. Currently, most treatment is directed towards individual diseases as they arise.

Development of new drugs to target ageing is a major challenge, thoughtfully addressed in the chapter by James Kirkland. Ageing is not recognized by regulatory authorities as a disease, or as a valid target of clinical trials, which would be prohibitively expensive because they would need to be of long duration, and to use initially diseasefree participants. It would be more feasible to test drugs that have been approved for specific diseases, and that also target mechanisms of ageing, against other age-related

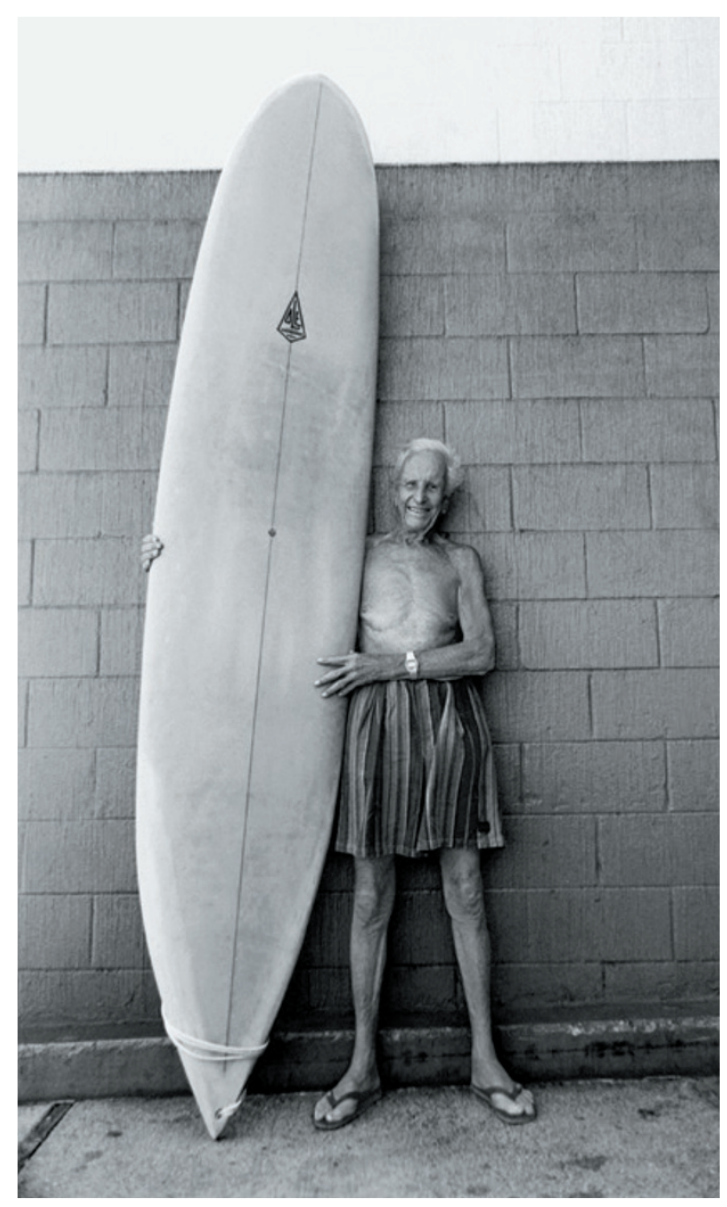

Woody, age 83.

conditions. For instance, older people tend to have a poor immune response to immunization against influenza; that response can be improved by pre-treating them with sirolimus, a drug already shown to increase animal lifespan and licensed as an immunosuppressant for use after kidney transplants. Polymorbidity (the presence of multiple conditions at once) has not yet been assessed as a potential drug target, and would require new, combinatorial measures of outcome and trials with older people, who are currently generally excluded from clinical trials. A 'polypill' of drugs that target
Aging: The Longevity Dividend EDITED BY S. JAY OLSHANSKY, GEORGE M. MARTIN AND JAMES L. KIRKLAND Cold Spring Harbor Laboratory Press: 2015.

different axes of ageing may give maximum protection, posing further interesting challenges for trials.

Olshansky discusses the ethics of targeting ageing for disease prevention. Ageing, he shows, is not nature's way of making space for the young; rather, it is a haphazard process of decline. The discovery that it is malleable allows an entirely new approach to improving the health and welfare of older people. Lifespan might increase slightly as a result, but the crucial point is that morbidity could be compressed. Olshansky also shows how the conventional route of treating single diseases could, perversely, increase overall morbidity, because the longer people live, the greater the part played by ageing in health status. Interfering in mechanisms of ageing provides the current best prospect for preventing cancer, cardiovascular disease and dementia.

From a health-economics perspective, examined by Dana Goldman, the results of reducing morbidity in older people would depend on public policy. If older people were healthier and more active, then they would depend less on others and would produce greater economic activity - from work and volunteering - both of which would be a net benefit to society. A direct comparison of the predicted economic consequences of the status quo, delayed cancer, delayed heart disease and delayed ageing showed that this last scenario would result in a much higher proportion of able older people. However, for that to create an economic benefit, age-related entitlements to various forms of support, particularly pensions, would have to change.

Basic science and human demographic studies have delivered an unprecedented opportunity to tackle the comorbidities of later life. To translate these discoveries into drugs and changes in medical practice, and to reap the consequent economic benefits, will require some radical changes: breaking down disease siloes, training a new generation of physicians and scientists capable of working across disciplinary boundaries, and altering public attitudes and policy.

Linda Partridge is founding director of the Max Planck Institute for Biology of Ageing in Cologne, Germany, and director of the Institute of Healthy Ageing at University College London. e-mail: linda.partridge@age.mpg.de 